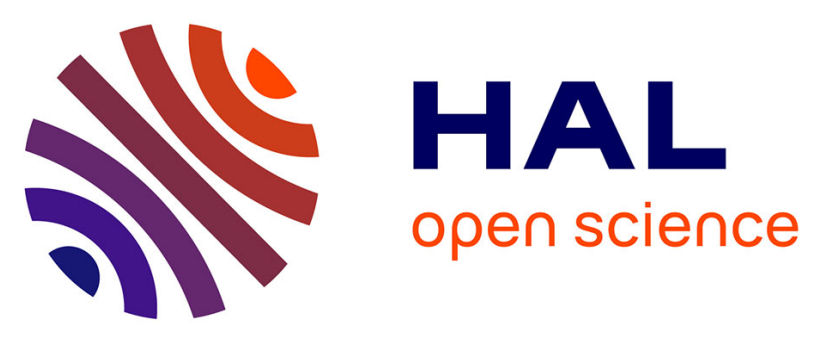

\title{
Le travail pour tous: une recherche-action en collectivité territoriale
}

Malika Litim, Dominique Lhuilier, Anne-Marie Waser

\section{To cite this version:}

Malika Litim, Dominique Lhuilier, Anne-Marie Waser. Le travail pour tous: une recherche-action en collectivité territoriale. Psychologie du travail et des organisations, 2019, 25, pp.40 - 53 . 10.1016/j.pto.2018.05.002 . hal-03486399

\section{HAL Id: hal-03486399 \\ https://hal.science/hal-03486399}

Submitted on 20 Dec 2021

HAL is a multi-disciplinary open access archive for the deposit and dissemination of scientific research documents, whether they are published or not. The documents may come from teaching and research institutions in France or abroad, or from public or private research centers.
L'archive ouverte pluridisciplinaire HAL, est destinée au dépôt et à la diffusion de documents scientifiques de niveau recherche, publiés ou non, émanant des établissements d'enseignement et de recherche français ou étrangers, des laboratoires publics ou privés.

\section{다)(1) $(5$}

Distributed under a Creative Commons Attribution - NonCommercial| 4.0 International 
Version of Record: https://www.sciencedirect.com/science/article/pii/S1420253018300220

Manuscript_e48bec3f2a324bb05d0366727fbf4e7a

\section{Le travail pour tous : une recherche-action en collectivité territoriale}

Malika Litim, UTRPP (EA 4403), Université Paris 13 Sorbonne Paris Cité, Villetaneuse, France, membre associée de l'équipe Psychologie du travail et clinique de l'activité, CRTD, Cnam, 06 711007 89, malika.litim@univ-paris13.fr.

Dominique Lhuilier, Centre de Recherche sur le Travail et le Développement (CRTD), Cnam.

Anne-Marie Waser, Laboratoire Interdisciplinaire pour la Sociologie Économique (LISE, UMR 3320), Cnam. 


\section{Résumé :}

La progression des inaptitudes médicales et demandes de reclassement liées aux importantes transformations du monde du travail a conduit à la mise en œuvre d'une recherche-action dans une collectivité territoriale. La méthodologie repose sur l'analyse de l'activité et les cadres théoriques de la clinique du travail. Les résultats concernent ici les dynamiques du reclassement: le reclassement "informel " et ses conditions, signe d'une relative plasticité de l'organisation du travail, et le reclassement tel qu'institué. Cette recherche-action a permis de découvrir des ressources collectives insoupçonnées, de transformer les différents dispositifs existants et de favoriser le travail pour tous, en bonne santé ou avec une santé altérée.

\section{Abstract :}

In the present french situation, there is an increase in inaptitudes and in the requests for occupational reclassifications. These are linked to the transformations that are affecting the world of work and have led to the undertaking of an action-research within a territorial community.

The methodology is based on the analysis of the activity and the theoretical resources of the work clinical approach. The results enlight on one side, the " informal " reclassifications and their conditions, which is the sign of a relative plasticity of the work organisation, on the other one the reclassification as it is institutionalized. The action-research has allowed to discover unpredictable collective ressources and to facilitate the work for all, whether healthy or unhealthy.

\section{Mots clés :}

Reclassement, inaptitude, santé, pénibilité, recherche-action

\section{Keywords :}

Occupational reclassification, inaptitude, health, penibility, action research 


\section{Introduction}

D'importantes transformations affectent le monde du travail et les salariés et se traduisent par une progression des absences pour raison de santé, un nombre croissant du nombre de personnes en usure professionnelle et du volume des restrictions d'aptitude et des inaptitudes ainsi que des demandes de reclassements. Ces évolutions préoccupantes peuvent être expliquées par divers facteurs : la prolongation de la vie active, avec le recul progressif de l'âge de départ à la retraite et donc le vieillissement des travailleurs, l'augmentation du nombre de personnes qui vivent et travaillent avec une santé fragilisée (Lhuilier \& Waser, 2016), les transformations qui accroissent la pénibilité du travail, notamment l'intensification, la précarisation et l'individualisation du travail. Ces changements complexifient aussi le maintien en emploi de nombre de salariés à la santé fragilisée.

Ces contraintes comme les ressources pour y faire face ne sont pas de même nature dans le secteur privé et dans la fonction publique. Dans le secteur privé, la grande majorité des salariés inaptes sont licenciés, alors que ceux-ci sont maintenus en emploi (mais pas nécessairement en activité) dans la fonction publique ${ }^{1}$. Mais les ressources antérieurement mobilisées pour ce maintien en emploi se réduisent drastiquement. Et dans un contexte de masse salariale contrainte, la file active des demandes de reclassement ne cesse de croître.

La montée en puissance de cette problématique dans la fonction publique, et notamment dans une collectivité territoriale confrontée aux impasses du procès de reclassement ${ }^{2}$, nous a conduit à engager une recherche-action (RA). Cette RA vise plusieurs objectifs: l'exploration et la transformation des conditions de maintien en activité, l'investigation des dispositifs formels existants et utilisés, mais aussi des processus plus informels qui président aux régulations individuelles, collectives, organisationnelles qui préviennent les processus de relégation, voire d'exclusion, et enfin, l'élaboration dans des dispositifs collectifs de propositions visant à la prévention de l'inemployabilité.

Nous présenterons dans un premier temps la construction de la problématique retenue ainsi que la méthodologie qui fonde cette RA. Puis nous retracerons les processus associés à l'impact d'une santé dégradée sur l'activité professionnelle individuelle et collective ainsi que les modalités et impacts de l'entrée dans la procédure de reclassement suite à un avis

\footnotetext{
${ }^{1}$ Il n'y a pas à ce jour de chiffres officiels consolidés au plan national sur le devenir des salariés « en inaptitude à tout poste ". Mais des enquêtes régionales comme celle réalisée par la DIRECCTE Aquitaine en 2015 donne des indications sur cette question dans le secteur privé. Elle souligne l'augmentation de $29 \%$ des décisions d'inaptitudes à tout poste dans le régime général de 2006 à 2014. Les pathologies à l'origine de l'inaptitude sont les TMS (44\%), les troubles mentaux (33\%), les autres maladies chroniques. Quant au devenir de ces salariés, $39 \%$ sont licenciés sans projet ni solution, $27 \%$ sont mis en invalidité, $26 \%$ sont licenciés avec un projet identifié et $6 \%$ sont mis à la retraite. Pour ce qui concerne le secteur public, un rapport de l'IGAS et de l'IGA indique qu' " aucune donnée chiffrée significative n'a pu être réunie sur les éventuelles pratiques de licenciement pour inaptitude pour raison de santé (...) De nombreuses situations ne sont traitées que par le recours à la position dite de "disponibilité d'office", pis-aller qui ne peut que favoriser une désinsertion professionnelle grave de l'agent » (Krynen et Yeni, 2011, p. 4).

${ }^{2}$ Cette recherche-action a été conduite par les auteurs, ainsi que Julie Cochin, psychologue, Sarah Lainé, conseillère en reclassement et Emilie Rerolle, psychologue.
} 
médical d'inaptitude.

\section{La pénibilité et le reclassement, deux enjeux majeurs de la fonction publique territoriale}

\subsection{Pénibilité, usure, santé fragilisée et marges de manœuvre}

La notion de pénibilité est ancienne : depuis la naissance de la médecine du travail en 1946, elle renvoie à la prise en compte des facteurs de risques professionnels et à la notion de charge de travail. Sa définition légale est beaucoup plus récente: elle s'inscrit dans des débats sur l'avenir et la justice du système de retraite. Aussi, elle constitue une construction à la fois sociale et politique qui trouve ses déclinaisons dans les différentes collectivités territoriales (CNFPT, 2014). Aussi, ici, nous nous référerons aux trois acceptions de la pénibilité telles qu'analysées par S. Volkoff (2015): celle qui repose sur une approche probabiliste, fondée sur des résultats épidémiologiques mettant en évidence les corrélations entre nuisances et prévalence des pathologies; celle qui correspond à un travail vécu comme pénible; et enfin, celle éprouvée par les salariés ayant des problèmes de santé qui leur rendent les exigences du travail peu supportables. Cette dernière dimension doit être soulignée, à la fois du fait de l'augmentation du nombre de salariés à la santé altérée et au vu des risques d'une usure professionnelle majorée.

Le caractère plus ou moins gênant de ces troubles dépend bien sûr de leur nature et gravité mais aussi, et plus fondamentalement, des exigences du travail et des marges de liberté dont les opérateurs disposent dans leurs situations de travail pour pouvoir tenir ensemble travail productif et travail de santé. Ce "travail de santé " recouvre des activités de soin de soi au double sens du cure et du care, des auto-prescriptions réglant le style et l'hygiène de vie, la réorganisation des actes exigés par la prescription médicale afin de les ajuster aux contraintes des différentes sphères de vie comme aux désirs et aspirations personnels, la réorganisation des tâches professionnelles, l'invention de nouvelles manières de faire pour construire des compromis entre exigences du milieu de travail et exigences de santé (Lhuilier \& Waser, 2016).

Les problèmes de santé n'impliquent pas mécaniquement des difficultés dans le travail (Jolivet \& Molinié, 2006), mais le défaut de marges de liberté permettant ou non un travail de santé peut à la fois les aggraver (jusqu'à " l'inaptitude » et la demande de reclassement) et être un puissant facteur de développement de l'usure prématurée au travail.

L'usure professionnelle est « un processus dynamique dans lequel entre en jeu de multiples déterminants qu'ils soient intrinsèques (genre, âge, catégorie socioprofessionnelle, parcours professionnel...) et extrinsèques (organisationnels, exposition à des agents et des situations de travail pathogènes...), qui se combinent et se cumulent au fil de l'existence en conduisant à des altérations à la santé des travailleurs dans une ou plusieurs de ses dimensions» (Pommier, Bardouillet, Gilles \& Molinié, 2006 ; Roux \& Volkoff, 2010). Elle se signale par des difficultés accrues à répondre aux exigences de la tâche et les médecins du travail sont de plus en plus confrontés aujourd'hui à " ceux qui n'en peuvent plus " (Lecomte-Ménahès, 2014). L'analyse de ces difficultés et des formes prises par le travail de santé permet de repérer les processus en amont des recours à l'inaptitude ou aux restrictions d'aptitude. Un certain nombre d'ajustements, d'arrangements, de régulations individuelles (Leplat, 2006), collectives et organisationnelles (de Terssac, 1992) sont développés. Enfin, l'importante 
variation interindividuelle dans l'expression et les manifestations d'usure rend plus aiguë la question de sa prise en charge dans l'organisation. Ici, l'encadrement de proximité peut favoriser les régulations collectives et jouer un rôle de détection et de prévention afin de maintenir la continuité du service et de s'épargner une charge d'organisation supplémentaire en cas d'absentéisme massif, entendu ici comme " mécanisme régulateur intervenant avant l'épuisement " (Veil, 2012, p. 151). Ce qui suppose qu'il puisse trouver les arrangements nécessaires pour l'organisation du service et la préservation des plus fragilisés (Haïlé-Fida, 2005).

En ce sens, la prévention de l'usure précoce est indissociable de la recherche des différentes voies visant à réparer et à compenser (Héas, 2010), qu'il s'agisse des aménagements de poste ou du reclassement des agents en inaptitude.

\subsection{L'inaptitude}

Chaque année en France, plus d'un million de salariés se voient notifier des avis de médecins du travail comportant des restrictions d'aptitude ou des demandes d'aménagement de poste de travail et plusieurs dizaines de milliers d'entre eux sont déclarés inaptes à tout poste de travail dans l'entreprise et licenciés ${ }^{3}$ (DIRECCTE Aquitaine, 2015 ; DIRRECTE Pays de Loire, 2012 ; DARES, 2015). Les questions relatives à l'inaptitude gagnent en importance et en complexité du fait des transformations relatives à la santé (développement du nombre de maladies chroniques ${ }^{4}$, vieillissement de la population active, meilleure prise en compte de la santé mentale...) et aux transformations des systèmes de travail.

"Si le salarié est inapte, c'est bien sûr parce que ses capacités physiques ou psychiques se sont dégradées, mais tout autant parce que l'environnement social du travail se révèle inadapté à son nouvel état de santé »(Le Bianic, 2003, p. 56).

Plus que le résultat d'une pathologie médicale, l'inaptitude est l'aboutissement d'un processus d'exclusion déjà amorcé pour d'autres raisons (rupture dans les régulations collectives, difficultés d'adaptation à un nouvel environnement de travail ou de nouvelles tâches, relations conflictuelles avec les collègues et/ou la hiérarchie...) (Laé, 1991).

\subsection{Dans la fonction publique}

La fonction publique est un environnement particulièrement propice à la montée des préoccupations relatives aux pénibilités du travail, inaptitudes et demandes de reclassement. En effet, les accidents du travail, l'absentéisme pour raison de santé, le nombre de maladies professionnelles reconnues (notamment les TMS qui constituent 80 à $90 \%$ des affections d'origine professionnelle) y sont en augmentation. Ces signaux sont mis

\footnotetext{
${ }^{3}$ II n'existe pas de chiffres officiels consolidés au plan national quant au nombre d'avis d'inaptitude à tout poste dans l'entreprise. Mais une estimation de l'AGEFIPH mentionnait 75000 cas pour l'année 2003, 58000 cas en 2001 et 28000 cas en1998.

${ }^{4}$ Les maladies chroniques constituent aujourd'hui les pathologies dominantes dans nos sociétés. Elles toucheraient, selon les estimations épidémiologiques disponibles, 15 millions de personnes atteintes plus ou moins sévèrement, soit $20 \%$ de la population française. Et leur nombre s'accroît régulièrement, principalement $\mathrm{du}$ fait des progrès thérapeutiques et du vieillissement de la population (Obrecht \& Hittinger-Le Gros, 2010).
} 
en perspective avec la réduction des marges de manœuvres budgétaires liée à la révision générale des politiques publiques. Celle-ci produit une réduction des recrutements et du turn-over générationnel normalement lié aux départs à la retraite (Krynen \& Yeni, 2011). Cette tension dans les effectifs contribue à une intensification du travail, associée à une pression à la productivité plus forte.

Ce sont ces principales transformations qui contribuent à la mise en visibilité et à l'agenda de la gouvernance des organisations publiques, et particulièrement dans la fonction publique territoriale du fait d'une dégradation reconnue des conditions de travail, de la problématique des pénibilités (CNFPT, 2014) et du reclassement (FNCDG, 2015). Elles ont conduit une collectivité territoriale à s'engager avec une équipe de chercheurs du CRTDCnam dans une recherche-action visant à améliorer les conditions du maintien en activité des agents ayant une santé altérée et à développer dans le même temps la prévention de I'usure au travail.

\section{La recherche-action : méthodologie et dispositif}

L'enjeu est, donc, pour les commanditaires, de trouver une issue à la situation de saturation: de plus en plus d'agents en attente de reclassement et une grande partie sans activité, un service dédié débordé qui ne parvient pas à trouver de solutions pour tous les agents, ceux-ci étant considérés par les différents services de la collectivité comme des poids et non comme des ressources. Ces mêmes commanditaires, peinant, de l'intérieur, à trouver des solutions, ont misé sur le pilotage par une intervention extérieure. Ils avaient en effet fait le constat, à l'occasion d'une démarche de réduction de l'absentéisme et de la prévention des risques professionnels, que la collectivité échouait à passer d'un plan d'actions aux actions effectives. En choisissant de faire appel à des analystes du travail, compris dans ses diverses acceptions, de surcroît spécialisés sur la problématique travail et maladies chroniques, ils souhaitaient donc donner un autre destin à la démarche.

La clinique du travail a pour centre le sujet aux prises avec des situations concrètes de travail: subjectivité et activité sont au cœur des investigations théoriques et méthodologiques. Le travail est conçu comme une activité orientée à la fois vers le segment de réalité à transformer, vers autrui et vers le sujet lui-même. Cette activité est fondamentalement sociale, réalisée avec d'autres, pour d'autres, en fonction de règles et repères produits collectivement. L'activité peut être porteuse d'une dynamique de développement. En effet, elle n'est pas déterminée mécaniquement par son contexte car elle cherche à le transformer et à affranchir le sujet des contraintes de la situation.

Si, au cœur de l'intervention se tient l'analyse de l'activité, celle-ci doit s'entendre aux différents niveaux de l'organisation et entre ces niveaux, ce qui suppose d'intégrer à la démarche une perspective globale impliquant les différents métiers et positions hiérarchiques contribuant au travail d'organisation (Sarnin \& coll., 2012 ; Althaus \& ali, 2013 ; Petit \& Dugué, 2013).

La méthodologie de la recherche-action repose sur l'analyse de l'activité (Leplat, 2008 ; Dujarier \& al., 2016), les cadres théoriques croisés de la clinique du travail (Lhuilier, 2006), de la démarche inductive de la sociologie pragmatique (Bessy \& Chateauraynaud, 1995 ; Boltanski, 1990) et sur les principes de l'intervention psychosociologique (Dubost 1987 ; Mendel \& Prades, 2002 ; Lhuilier, 2017). 
L'analyse de l'activité, du travail réel, des métiers est au fondement des processus de transformation que cette RA cherche à développer. Elle a donc été conçue en trois phases distinctes, la première visant la co-construction d'un diagnostic sur la base duquel la deuxième phase est celle de l'action proprement dite. L'objectif étant qu'à terme les résultats s'inscrivent durablement dans l'organisation (troisième phase). Notons que cette dernière phase est encore en cours.

\subsection{La co-construction d'un diagnostic}

Cette première phase, qui a duré un an (initialement prévue pour une durée de 6 mois), a poursuivi plusieurs objectifs :

- Investiguer auprès de services (volontaires) dont l'activité était jugée pénible, usante ce qui rend difficile la réalisation des tâches et des missions et les solutions qui existent pour y faire face. Ont été retenus deux services des parcs et jardins, une équipe de restauration scolaire, deux EHPAD, l'équipe d'Atsem d'une école et une mairie de quartier. Ces investigations ont pris la forme d'observations et d'entretiens individuels et collectifs.

- Explorer, lors d'entretiens individuels avec des agents "reclassés " ou en " attente de reclassement " (volontaires), les divers processus à l'œuvre, des premiers éprouvés de la pénibilité à l'avis d'inaptitude en passant par les arrangements négociés au sein des équipes de travail.

- Inventorier les différentes ressources et obstacles organisationnels, lors d'entretiens avec des managers de différents services de la collectivité, les organisations syndicales et au sein du pôle $\mathrm{RH}$ avec le service en charge du procès de reclassement, la médecine de prévention, la mission Handicap.

Au total, ce n'est pas moins de 107 entretiens individuels, 17 entretiens collectifs et 28 observations qui ont permis de construire le diagnostic.

D'abord diagnostics locaux, ceux-ci ont fait l'objet de restitutions et de validation avant de constituer un diagnostic global présenté notamment au Comité de direction générale et au CHSCT.

\subsection{La phase des expérimentations}

A l'issue du diagnostic, c'est un dispositif d'ampleur qui a été mis en place, engageant un nombre important d'acteurs de la collectivité ${ }^{5}$, à travers la constitution de groupes de travail co-animés par un ou des membres de la collectivité et un intervenant-chercheur du Cnam. Les co-animateurs ont été réunis préalablement durant une journée pour une sensibilisation à l'analyse du travail.

La visée principale de cette phase était de proposer des expérimentations susceptibles de lever les freins identifiés dans différents secteurs et niveaux de la collectivité. Ces groupes ont été régulièrement réunis durant 7 mois.

Dispositif

${ }^{5}$ Au total, la RA aura mobilisé environ 300 personnes.
Nombre de

Nombre 


\begin{tabular}{|c|c|c|}
\hline & participants & $\begin{array}{c}\text { de } \\
\text { séances }\end{array}$ \\
\hline \multicolumn{3}{|l|}{$\begin{array}{l}\text { Trois « groupes métiers »: } \\
\text { Objectifs : échanger les expériences, explorer les stratégies individuelles et } \\
\text { collectives de maintien au travail, construire des accords en pratique sur } \\
\text { l'équité compte tenu des variations des capacités productives, les } \\
\text { ressources à mobiliser... }\end{array}$} \\
\hline - Groupe de jardiniers & 15 & 5 \\
\hline - Groupe restauration scolaire & 10 & 5 \\
\hline - Groupe crèche & 15 & 4 \\
\hline $\begin{array}{l}\text { Deux groupes « usagers du reclassement ": } \\
\text { Objectifs : sortir de l'isolement, collectiviser le travail de santé, échanger les } \\
\text { expériences, construire des stratégies de retour au travail, réfléchir aux } \\
\text { aménagements de poste et de manières de faire, s'informer sur les droits, } \\
\text { les ressources à mobiliser... }\end{array}$ & $\begin{array}{l}\text { G1 : } 12 \\
\text { G2 : } 11\end{array}$ & $\begin{array}{l}\mathrm{G} 1: 6 \\
\mathrm{G} 2: 6\end{array}$ \\
\hline \multicolumn{3}{|l|}{$\begin{array}{l}\text { Deux groupes "acteurs du reclassement » (services RH impliqués, } \\
\text { médecine préventive, encadrants de services...) } \\
\text { Objectifs généraux : développer la coopération entre les différents services } \\
\text { et acteurs, la circulation d'informations, produire des instruments de } \\
\text { pilotage, des outils et procédures utiles à l'amélioration du traitement des } \\
\text { situations }\end{array}$} \\
\hline $\begin{array}{l}\text { Groupe "prévention " } \\
\text { Objectifs: prévention primaire, proposer une méthodologie pour des } \\
\text { organisations du travail respectueuses de la santé, élaborer des outils } \\
\text { permettant un développement des carrières «fluide" }\end{array}$ & 11 & 5 \\
\hline $\begin{array}{l}\text { Groupe "suivi " } \\
\text { Objectifs : construire des outils permettant une meilleure prise en charge } \\
\text { des agents, soit dans le maintien dans leur emploi ou leurs souhaits de } \\
\text { mobilité, soit en attente de reclassement ou en cours de reclassement }\end{array}$ & 11 & 5 \\
\hline $\begin{array}{l}\text { Un groupe « directeurs et chefs de service » } \\
\text { Objectifs : analyser leurs propres activités en lien avec la prévention de la } \\
\text { fabrique de l'inemployabilité des agents, de l'usure prématurée pour } \\
\text { développer le travail pour tous : penser collectivement la régulation } \\
\text { d'objectifs contradictoires : réduction effectifs/réduction de la pénibilité de } \\
\text { certains métiers/ prévention de l'usure prématurée/accueil des agents } \\
\text { reclassés et/ou retour au travail suite à arrêts, outils de pilotage }\end{array}$ & 17 & 2 \\
\hline $\begin{array}{l}\text { Un groupe " syndicats" } \\
\text { Objectifs : analyser leurs propres activités pour développer les démarches } \\
\text { préventives et collectives, développer le rôle du CHSCT, des représentants } \\
\text { du personnel dans la prévention de l'inemployabilité et pour le travail pour } \\
\text { tous }\end{array}$ & 10 & 5 \\
\hline
\end{tabular}

Par ailleurs, une journée sur la problématique " prévention des pénibilités et maintien en emploi " a été organisée avec l'ensemble de l'encadrement de la collectivité, journée durant laquelle des ateliers de travail ont succédé la présentation du diagnostic. 
Tout au long du processus, un groupe "Projet " (composé de directeurs, de représentants de chacune des organisations syndicales, du pôle $\mathrm{RH}$ et des médecins de prévention) constitué comme instance de pilotage de la démarche, a examiné les propositions émanant des autres groupes et décidé des expérimentations à engager.

\section{Santé fragilisée et conditions du reclassement}

Parmi les premiers résultats significatifs de la RA, nous présenterons ici ceux qui concernent plus spécifiquement les dynamiques du reclassement: d'une part le reclassement " informel ", signe d'une relative plasticité de l'organisation du travail", et d'autre part, le reclassement, tel qu'il est institué, et à propos duquel il y a consensus sur son dysfonctionnement.

\subsection{Des régulations dans le cours du travail}

\subsubsection{Du reclassement officieux au reclassement institué : le cas du service espaces verts}

Au sein d'une équipe du service espaces verts, les douleurs au dos chroniques de deux jardiniers étaient suffisamment manifestes aux yeux de leurs collègues et connues du service de santé au travail pour que l'encadrement de proximité leur propose des tâches allégées mais utiles à l'équipe. Ils prennent l'initiative d'entreprendre d'autres menus travaux dans l'objectif de faciliter le travail de leurs collègues. Investissant l'entrepôt, lieu de stockage du matériel, ils ont commencé à recenser, ranger, classer, réparer les outils, affûter les lames, repeindre un local. Se dessinent ainsi les contours de ce qui deviendra le " magasin " dans lequel tout le matériel est entreposé, entretenu, comptabilisé afin de gérer le stock. Le service rendu est véritablement apprécié de tous : matériel toujours disponible, en état de fonctionner, référencé et attribué à une équipe pour la journée...

La reconnaissance de ces contributions se traduit par l'élaboration de deux fiches de poste produites par le directeur du service et la responsable RH. Après une période d'essai jugée positive de part et d'autre, le directeur a fait valider par sa hiérarchie et le service de prévention ces deux postes qui ne portent pas le label d'aménagés. L'un remplit des fonctions de menuisier. L'autre a une fonction de magasinier et participe progressivement à des tâches de ressources humaines (contrôle des présences, des retards des jardiniers, gestion des congés) et seconde à présent le directeur adjoint.

Ceux deux cas révèlent des "reclassements internes ", initialement informels puis institués : car les aménagements ont été élaborés, testés, réévalués en interne, entre jardiniers, encadrement de proximité, directeur du service, responsable $\mathrm{RH}$, puis validés in fine par les acteurs du reclassement. Plus largement, cette façon de procéder semble s'inscrire dans une tendance de ce service à prendre en compte le processus d'usure professionnelle, des jeunes et moins jeunes, en interne. Elle serait motivée par un relatif échec des reclassements externes à ce service (des jardiniers reclassés dans d'autres métiers

\footnotetext{
${ }^{6}$ Nous présentons ici seulement deux exemples des nombreux métiers (250) et domaine d'activités de la FP et donc de la Ville. Cette RA a conduit à des observations comparables en matière d'impact de marges de manœuvre contrastées pour réorganiser le travail afin de préserver la santé des agents et maintenir en activité ceux qui ont des problémes de santé dans les EHPAD, les crèches, les mairies de quartier...
} 
reviennent aux Parcs et Jardins), par une image du "reclassé " difficile à porter car synonyme de fragilité, manque ou perte de professionnalité et surtout par un souhait largement partagé par les agents de faire carrière dans ce métier notamment lorsqu'il a été choisi. Ce souhait se présente comme une véritable opportunité de transformation puisque pour permettre aux agents de faire carrière, tous doivent ensemble se soucier de pouvoir y travailler longtemps dans des conditions qui doivent évoluer au fil des âges (Molinié, Gaudart \& Pueyo, 2012). Les restructurations organisationnelles récentes et la façon dont les agents de maîtrise encadrent semblent y concourir avec un certain succès : les périmètres des secteurs sur lesquels interviennent les différentes équipes ont été en partie construits de façon à solliciter un " travail de précision " dans deux secteurs de la Ville et un travail plus en force, plus répétitif, dans les autres secteurs. Ainsi, les agents " usés ", des jardiniers avec une grande ancienneté dans ce service, se voient proposer de rejoindre une des équipes qui font du "travail de précision" au centre Ville. Celle-ci est considérée comme " une vitrine (...) il faut que ça soit beau devant les bâtiments historiques (...) on soigne les parterres (...) c'est l'image de la Ville ". Le travail est varié, moins physique et perçu comme valorisant par les anciens jardiniers qui ont, pour certains, une formation horticole qu'ils peuvent ainsi mettre en avant. Bien qu'aucun parcours au sein du service Parcs et Jardins ne soit conçu en amont pour les jardiniers qui manifestent des fragilités, la direction et l'encadrement intermédiaire semblent très vigilants au fait de conserver des marges structurelles et organisationnelles leur permettant de faire ce qui s'apparente fortement à du reclassement mais qui ne porte pas ce nom jugé péjoratif. Les jardiniers semblent s'accorder non seulement en principe mais aussi en acte : prévenir la pénibilité car lorsque les altérations (accidents ou usures) sont survenues dans le service Parcs et Jardin, il est de la responsabilité collective de proposer un travail soutenable, au sein du service, aux agents "victimes (...) c'est pas de leur faute, il faut bien qu'on leur propose quelque chose d'autre ". Comme nous le verrons plus loin, tous les agents " usés " de ce service ne sont pas logés à la même enseigne: certains semblent mériter plus que d'autres les postes "doux " et valorisants.

\subsubsection{L'impossible aménagement : le cas du service de la restauration scolaire}

Dans le service de restauration scolaire, le processus de reclassement interne semble être en panne. Alors que de nombreux agents travaillent avec des douleurs chroniques et un moral en berne, très peu ont recours à ce dispositif de reclassement car synonyme de perte d'une place qu'ils ont contribué à forger (entraide, relations avec le personnel éducatif, etc.).

Le reclassement semble bloqué dans les deux sens: les responsables de restaurants ne souhaitent pas accueillir d'agent reclassé dont ils ont une représentation négative : "il faut être en forme pour faire ce métier ", et les agents appréhendent l'épreuve du reclassement qui les conduirait nécessairement vers un métier déclassé à leurs yeux (" on n'est plus rien ») et où les marges de liberté seraient encore diminuées (" être assis à un bureau, on ne peut pas bouger, c'est pas pour moi »). Contrairement aux espaces verts, ce service ne parvient pas à identifier des tâches qui pourraient être réalisées par des agents dont les limitations fonctionnelles sont importantes ou chroniques. Travailler jusqu'au bout, jusqu'à ce qu'on ne puisse plus se lever le matin, semble être la ligne de conduite d'une majorité d'agents rencontrés qui tous disent aimer leur métier et ne pas vouloir en changer.

Lors d'observation du travail d'une équipe de cinq agents dans un restaurant qui sert 320 repas par jour, une auxiliaire de vie est présente. Son rôle est en principe de soutenir et aider l'agente revenue en restauration scolaire après une longue maladie. Or l'observation 
montre que l'auxiliaire de vie tient un poste à part entière qui certes soulage l'équipe, mais ne règle que partiellement la difficulté majeure éprouvée par l'agente: une fatigue chronique qui la conduit à travailler lentement. Pour tenir sa place dans l'équipe, elle souhaite participer à toutes les tâches et en particulier les moins nobles (nettoyage).

La responsable et l'équipe de ce restaurant ne sont pas parvenues, contrairement aux espaces verts, à proposer un poste sur mesure, utile et pérenne à cette agente afin qu'elle puisse non seulement se maintenir mais aussi retrouver de l'énergie.

L'organisation de ce service a été fondamentalement transformée avec la mise en place d'une cuisine centrale et le passage de tous les restaurants scolaires de la Ville en liaison froide. L'équipement neuf, conçu par des ergonomes, devait lever toutes les pénibilités. Une majorité des agents a cru à cette promesse qui les propulsait dans une modernité qui devait valoriser du même coup ce métier peu considéré par les agents de la sphère éducative et des parents d'élèves. Or plaintes et absences du personnel dans les restaurants sont en nette progression obligeant ainsi le recrutement d'intérimaires alors que cette réorganisation a été présentée aux élus et à la direction comme source de diminution de la masse salariale permettant par là de répondre aux exigences de la RGPP, les équipes des restaurants passant de 10 à 5 agents. A l'évidence, le fait qu'un bon nombre d'agents soit fragilisé par une carrière déjà longue n'a pas été pris en compte dans les calculs du quota : nombre de repas servis/nombre d'agents par restaurant. Rythmes de travail, nouvelles tâches et relations au sein de l'équipe viennent percuter des façons de travailler qui fonctionnaient du temps " où on faisait la popote ensemble (...) où on pouvait se parler » et contenaient tout un ensemble de régulations. Depuis la liaison froide, l'équipe est composée d'un agent par fonction alors que précédemment, avec une équipe plus nombreuse, plusieurs agents couvraient le même poste. Cette nouvelle organisation, sans marge, exerce de fortes pressions " on est à bloc tous les jours " et, lors d'une baisse de forme ou de l'absence d'un collègue, la tenue des objectifs relève de la prouesse: "quand on est à l'auto-laveuse, on ne peut pas être au service en même temps (...) alors soit on ne lave pas les assiettes pour le second service, soit on fait déjeuner les enfants à $13 \mathrm{~h}$ au lieu de $12 \mathrm{~h} 30$ (....) mais c'est impossible ". Cette pression temporelle quotidienne (servir 200 à 400 repas à heure fixe) associée à une vision idéalisée d'une cuisine centrale qui diminuerait mécaniquement toutes les pénibilités rend quasi impossibles tous les aménagements, régulations, reclassements en interne comme observés dans le service des Parcs et Jardins.

\subsection{Reclassement ou déclassement}

Du côté des "acteurs du reclassement", le constat du procès en panne est largement partagé :

- L'accent mis sur la réparation laisse en suspend la question de la prévention des inaptitudes.

- Les parcours professionnels ne sont pas pensés comme évolutifs, permettant des réorientations ou reconversions professionnelles en cours de carrière. Le peu de mobilité professionnelle tient à la fois à une faible visibilité des métiers alternatifs, à un accès à la formation ordonné aux "besoins du service " et non à la mobilité préventive dans les métiers pourtant reconnus comme usants. 
- Le service dédié à l'accompagnement au reclassement, à la réintégration des agents suite à un CLM, un CLD ou d'une mise en disponibilité, est débordé par le nombre $d^{\prime}$ agents ${ }^{7}$ à accompagner et se heurte à divers obstacles. Ceux-ci sont les fruits des politiques passée (la Ville a embauché massivement des agents de catégorie $C$, souvent dans des services dont l'activité est pénible, sans penser à leur employabilité à long terme) et présente (la performance attendue dans des contextes de "masse salariale maitrisée ", les rend réticents à prendre sur des postes pérennes les agents à reclasser perçus comme déficients);

- Les médecins de prévention doivent en supporter les dilemmes, par exemple en décidant de maintenir en arrêt un agent, contre l'avis du médecin traitant, parce qu'aucun aménagement de poste ou reclassement n'est possible sans " mettre le feu " aux services déjà en tension ;

- Les réunions pluridisciplinaires (service d'accompagnement, mission $\mathrm{RH}$, service conditions de travail, médecins de prévention...), dont la vocation était de résoudre les cas les plus difficiles ont fini par être désertées tant elles échouaient à résoudre les problèmes.

D'où le verdict de ceux qui en ont fait ou font encore l'expérience: "Dès que la médecine du travail dit "je vous mets en reclassement ", c'est fini ", "ça a été comme une sanction", " on a l'impression de mourir à ce moment là ». D'ailleurs, excepté le contact avec le service dédié à la protection sociale pour les aspects administratifs, les agents n'ont plus de contact avec la Ville, et en particulier les $\mathrm{RH}$, qui de leur côté supposent qu'il vaut mieux ne pas les déranger. A moins qu'il s'agisse en fait de les éviter.

Certains peuvent ainsi rester des années sans aucune opportunité de reclassement, et à la maladie s'ajoute le délitement des rapports sociaux et les difficultés à se projeter dans " une nouvelle vie professionnelle ": "Je suis chez moi, payé à rien faire et ça, je vous assure c'est très dur parce qu'après, le contact avec les gens à l'extérieur, j'ai du mal " "Moi c'est pareil, je ne sortais plus"; "j'ai l'impression de ne plus savoir rien faire, je n'ai plus confiance en moi ».

Les agents en arrêt finissent par connaître des difficultés financières (perte de primes, dépenses de santé, demande de remboursement quand l'imputabilité n'est pas reconnue, délais de la prise en charge par la prévoyance...) qui radicalisent leur sentiment d'injustice et d'abandon: " c'est le reflet de la société, on n'existe plus quand on n'est pas productif ". Ils s'interrogent sur les critères de sélection des " urgences " dans la file active des demandes de reclassement. Le recours aux organisations syndicales semble alors la principale voie de sortie de l'oubli. "Un coup de fil de temps en temps, ça montre qu'on est toujours vivant, qu'on existe ", "c'est dur d'être traité comme ça, on ne comprend pas pourquoi on a mérité ça... ».

Le reclassement leur apparaît donc comme une double peine, un déclassement, une forme de sanction douce au sens où elle n'est pas l'occasion d'une reconversion professionnelle mais plutôt d'une mutation sur un poste sans qualité, bordée de marques d'incompétence. Le deuil du métier est d'autant plus éprouvant qu'il est sans projection possible sur des alternatives. Le poids du stigmate associé à la catégorisation "reclassé " se prolonge dans celles associés aux enclaves à reclassés, là où ils se retrouvent le plus souvent affectés

\footnotetext{
${ }^{7}$ environ 400 sur un effectif global de 4500 agents dans cette collectivité.
} 
(cimetières, musées, zoo, service Etat civil ou en surnuméraires au service RH...). Le reclassement semble essentiellement pensé au prisme de l'emploi plus que de l'activité. Si les agents ne perdent pas leur statut, ils sont sur des durées de plus en plus longues en situation d'inactivité forcée (il peut leur être demandé de faire prolonger leur congé maladie, ou sont "mis en disponibilité d'office " ou au "placard»). Et les modalités de retour au travail s'attachent plus au repérage des emplois potentiellement ouverts ou aux affectations en renfort sur des postes administratifs qu'à un accompagnement anticipé des transitions professionnelles, étayé sur un bilan de compétences et la construction d'un projet de reconversion.

Aussi, le parcours vers la réaffectation apparaît comme semé d'embûches, alimentant l'anxiété d'être dessaisi de son avenir professionnel, de n'être qu' « un pion déplacé ».

\section{Discussion: les conditions du travail pour tous}

\subsection{Etre acteur de son maintien en activité grâce aux régulations et accords sur les principes de justice}

La comparaison entre le secteur des espaces verts et de la restauration scolaire permet d'identifier les conditions autorisant, à effectif constant, des aménagements débouchant sur des postes de reclassement interne valorisés par tous et les principes de justice qui sont mobilisés pour valider la division du travail.

Plusieurs types de conditions apparaissent primordiales pour permettre le reclassement. En premier lieu, comme souvent souligné par de nombreux travaux (Caroly, Simonet, Vezina, 2015 ; Coutarel 2005 ; Gollac et Volkoff, 2000), les marges de manœuvre dont disposent les agents et les équipes sont essentielles pour faire face aux aléas (absence d'un collègue, météo...) et aux variations des capacités productives individuelles.

Dans les espaces verts, les objectifs sont définis à l'échelle de la semaine. Ainsi s'il pleut lundi, la tonte prévue ce jour est remise au lendemain. Alors que dans les cuisines, les repas doivent être impérativement servis chaque jour à heure fixe. De plus, depuis la mise en place de la cuisine centrale, le personnel dans chaque restaurant a fortement été réduit et chacun est affecté à une fonction précise (la plonge, le service...). La rationalisation du processus du service de restauration a fait fondre les marges et donc les régulations permettant de faire face aux aléas.

Tout s'enchaîne: peu d'autonomie, peu de régulations et un collectif éclaté dans des fonctions ne permettant guère l'entraide dans les restaurants alors qu'elle est fortement mobilisée dans les espaces verts. Quand ces marges sont préservées et autorisent des aménagements informels, ceux-ci sont l'occasion d'une réorganisation du travail de l'ensemble du service, d'une sollicitation de compétences organisationnelles (Gaillard \& de Terssac, 2013 ; de Terssac, 2008).

La deuxième condition, rarement évoquée dans la littérature scientifique, est celle de la réévaluation des principes de justice mobilisés par les agents pour établir des équivalences entre eux, entre les objets (outils), entre les tâches (Boltanski \& Thévenot, 1991 ; Boltanski, 1990). Les réorganisations du travail modifient ces équivalences puisque toutes les tâches ne se valent pas et tous les agents ne peuvent participer à toutes les tâches ou s'ils font les 
mêmes tâches, ils ne les font pas au même rythme. De même comme souligné précédemment, tous les agents ne se valent pas au sens où certains vont bénéficier plus que d'autres des aménagements informels réalisés dans les équipes.

Initialement, le soupçon de fainéantise, de mauvaise foi pèse souvent. II contribue à dessiner deux clans: celui des fainéants et celui des bosseurs. Dodier (1986) a montré que les questions de santé sont rejugées sur la place du travail. Les dispositifs médicauxadministratifs (arrêt de travail, restriction d'aptitude, inaptitude, invalidité) ne suffisent pas à légitimer l'aménagement. La validation de la réorganisation du travail au sein d'une équipe semble nécessairement passer par une réévaluation des principes de justice permettant une redéfinition des équivalences entre tâches, agents. Le cas des deux jardiniers témoigne des conditions des accords: reconnaissance des causes des altérations fonctionnelles, des compétences professionnelles et des qualités humaines. Un accord au nom du principe du mérite (Dubet, 2006, p. 90) est alors possible. Et ce d'autant plus que les postes aménagés rendent service à tous. Cette acceptation s'inscrit donc aussi dans une logique de sollicitude et réciprocité (Huyez-Levrat \& Waser, 2014).

A contrario, quand l'interconnaissance, et donc la reconnaissance, ne sont pas suffisantes, ce sont les principes d'égalité qui prévalent. Ils ont de grandes chances de mettre l'agent à la santé fragilisée en difficulté. Alors, l'octroi de restrictions d'aptitude tend encore plus les relations car éveille le soupçon notamment dans les cas où les problèmes de santé ou le handicap sont invisibles, indicibles.

\subsection{Etre acteur de son reclassement par l'entremise du collectif}

Du coté des agents inscrits dans le processus institué de reclassement, l'épreuve de la solitude, de l'amertume, voire du ressentiment, mais aussi le défaut de ressources collectives susceptibles de favoriser la construction de projets professionnels alternatifs, est manifeste. Les dispositifs d'accompagnement individualisent et renforcent ces épreuves. Ils accroissent la durée du processus et l'anxiété.

L'obligation de reclassement (Desbarat, 2010 ; Fantoni, 2016) se traduit le plus souvent par une gestion au cas par cas, peu anticipée et où prévaut la dimension fortement discrétionnaire du processus à l'œuvre (reconnaissance des qualités professionnelles, degré de proximité relationnelle entre le salarié et son équipe) (Maresca \& Dujin, 2011). De plus, " paradoxalement, entrer en inaptitude réclame de bonnes capacités d'adaptation: gérer l'incertitude de sa situation, s'adapter à un nouveau métier, à une nouvelle équipe " (Laé, 1991). Pour être apte à un emploi d'inapte, il faut à la fois satisfaire aux attentes professionnelles (répondre aux règles du métier) et à l'attente médicale (répondre aux défaillances présupposées par ce classement); ce qui installe, souvent durablement, dans ces zones de relégation que sont les prolongations de l'arrêt maladie, ou les " placards pour inaptes " (Lhuilier, 2002). L'incertitude pèse de tout son poids sur "l'inapte " suspendu à une décision à venir. Et nos observations rejoignent les nombreux travaux qui soulignent l'importance de la dimension temporelle dans le processus de retour au travail, "réadaptation ", reclassement comme la nécessité de prévenir l'installation dans la longue durée de ces périodes d'attente et de retrait de l'activité (Baril, 2002 ; Fantoni-Quinton \& Frimat, 2011 ; Mezza, 2017). 
Mais au delà de cette dynamique temporelle, nous souhaitons mettre l'accent ici sur l'apport de dispositifs collectifs et non pas seulement individuels dans cet accompagnement aux transitions professionnelles. Notre expérimentation de dispositifs collectifs d'agents reclassés ou en attente de reclassement permet de repérer les ressources puisées dans ces groupes : chacun a pu réaliser qu'il n'était pas seul à vivre cette épreuve et contribuer à un échange d'expériences entre pairs, au partage des informations indispensables pour " être acteur $»$ de son reclassement.

La méconnaissance des rouages institutionnels, tout comme l'incompréhension des courriers des experts, sont autant d'éléments qui favorisent le sentiment d'abandon. Face au dédale des procédures, s'est construit dans ces échanges une sorte de cahier des charges d'un " guide du reclassement » en cours de réalisation.

Ont aussi été explorés lors des réunions, des stratégies pour investiguer le champ des possibles grâce à l'activité réflexive (s'interroger sur ce qu'on aimerait faire, sur ses compétences et désirs) à la faveur des informations données par la co-animatrice interne ou encore des arguments pour arbitrer entre le dire ou taire sa maladie. L'occasion offerte leur a permis se "remettre en selle ", en restaurant leur confiance en eux par les discussions à propos de leur situation individuelle.

Ces échanges permettent de déprivatiser l'expérience de la maladie au travail pour en faire un objet commun autour duquel rencontrer les autres. Ils tendent à inverser le rapport à cette condition subie d' " agent en reclassement " pour restaurer les capacités d'action sur celle-ci: un passage peut alors s'opérer entre des attentes de prise en charge à une mobilisation pour la construction de sa propre transition professionnelle (Orly-Louis \& al., 2017).

On retrouve ici les fonctions de dispositifs collectifs permettant d'être acteur de sa trajectoire professionnelle (Lhuilier \& al., 2014) : c'est précisément la ressource collective qui est apparue comme le résultat le plus précieux de cette expérimentation. Aussi il a été proposé et retenu que ce type de groupe soit dorénavant institué. A partir du bilan tiré des 2 groupes expérimentaux d'agents en attente de reclassement ou en arrêt longue maladie, l'instauration de groupes dits "passerelle" va se développer. Le premier mis en place concerne des personnels travaillant en crèche et reconnus comme " inaptes " : I'exploration collective du champ des possibles au sein de la Ville va de paire avec l'élaboration partagée du deuil d'un métier perçu par tous comme particulièrement usant dans la longue durée d'une vie professionnelle.

\section{Conclusion}

Cette RA rejoint des travaux précédents qui développent une approche des relations "santé-travail " au fil de l'âge et des parcours professionnels. Les dimensions processuelles et diachroniques de ces relations sont éclairées à partir des travaux sur le vieillissement. Trois registres complémentaire sont distingués: (1) le vieillissement "par » le travail considérant les empreintes du parcours professionnel antérieur sur la santé ; (2) le vieillissement "par rapport " au travail examinant les processus de sélection liés à l'âge particulièrement, sous l'effet des conditions de travail et renvoyant notamment aux inégalités sociales; ( 3 ) le vieillissement " dans " le travail s'intéressant aux phénomènes de régulation en inscrivant la santé en situation réelle de travail par l'élaboration de compromis opératoires entre la tâche et les capacités des individus eu égard des marges de manœuvre 
qui leur sont laissées, leur âge et leur expérience (Molinié \& Pueyo, 2012).

L'avancée en âge étant un processus de changement continu, et donc non réductible à la thématique des "seniors" dans le monde du travail aujourd'hui, on peut constater un accroissement des traces négatives du travail sur la santé (usure précoce et accroissement des avis d'inaptitude), une plus grande vulnérabilité sociale des agents en catégorie C, exerçant des fonctions dites d'exécution, et une amplification des processus de relégation jusqu'à l'entrée dans le procès de reclassement lorsque l'organisation du travail se rigidifie et contrecarre les stratégies de régulation et de compensation déployées pour tenir ensemble la dialectique travail, emploi et santé.

La prévention de la désinsertion professionnelle suppose d'intégrer cette perspective diachronique et de restaurer la continuité entre la prévention primaire des accidents et maladies d'origine professionnelle, la prévention secondaire face aux troubles infrapathologiques ou dès l'apparition des problèmes de santé avérés, et enfin tertiaire, pour réduire les effets sur l'emploi et sur l'activité d'une santé dégradée. "Ce séquençage dans le temps introduit également la notion de parcours : parcours professionnel tout d'abord où il s'agit de suivre un salarié tout au long de sa vie professionnelle et quels que soient les statuts professionnels, la nature des contrats et leurs éventuelles ruptures; mais aussi parcours personnel couvrant plus de 45 ans de vie à l'âge adulte, parcours potentiellement émaillé d'évènements de santé, pathologies chroniques et survenue de handicaps, liés ou pas au travail, qui menacent l'insertion professionnelle et dont il convient d'éviter l'aggravation par le travail » (Aballéa \& Mesnil du Buisson, 2017, p. 13).

En visant l'amélioration des conditions du maintien en activité des agents, notre RA nous a conduit à explorer et comparer les ressources mobilisées ou empêchées au sein de services. Là où il y a des marges de manœuvre, la réorganisation du travail et la réévaluation des principes de justice favorisent le travail pour tous, en bonne santé ou avec une santé altérée. En s'intéressant plus précisément aux agents dont l'état de santé ne permet plus de poursuivre leur activité, et en prenant acte des limites de l'accompagnement individuel, cette recherche-action a permis de découvrir des ressources collectives insoupçonnées et être force de propositions pour améliorer les différents dispositifs existants ou en proposer de nouveaux. A titre d'exemples, un guide du reclassement à destination des agents et managers de la Ville est en cours d'élaboration, les mobilités pour raisons de santé sont examinées en priorité, un tutorat est institué pour les accompagner, différentes mesures sont prises pour maintenir le lien avec les agents durant leur arrêt maladie, la possibilité de temps d'immersion- découverte de métiers est généralisée, des rencontres sont organisées entre managers et médecins de prévention sur les avis de restrictions d'aptitude et inaptitudes, les visites de pré-reprises sont systématiquement proposées...

Cette RA entend souligner la nécessité de combiner deux champs d'actions: en matière d'accompagnement des transitions professionnelles marquées par des événements de santé (suite à un accident de travail, une maladie, un handicap) et en matière de développement d'un travail soutenable. A cette condition, le travail pour tous sera favorisé. A ce stade, la collectivité et en particulier ses services $\mathrm{RH}$ et ses directions opérationnelles, doivent poursuivre les transformations, en refondant le procès du reclassement et en favorisant des organisations respectueuses des variabilités individuelles. Enfin, un travail en cours avec le CNFPT doit permettre que les enseignements de cette RA sur ses deux volets, prévention de I'usure professionnelle et accompagnement aux transitions professionnelles, soient transmis via la construction d'un MOOC, formation en ligne ouverte à l'ensemble des acteurs de la 
FPT.

Les auteures déclarent ne pas avoir de lien d'intérêts.

\section{Références bibliographiques}

Aballéa, P., \& Mesnil du Buisson, M.-A. (2017). La prévention de la désinsertion professionnelle des salariés malades ou handicapés, Rapport de l'IGAS, Tome 1.

Althaus, V., Grosjean, V., \& Brangier, E. (2013). La centration sur le processus du changement : I'apport de l'intervention systémique à l'amélioration du bien-être au travail. Activités, 10-1, 127-149, http://activites.revues.org/607; DOI : 10.4000/activites.607.

Baril, R. (2002). Du constat à l'action : 15 ans de recherche en réinsertion professionnelle des travailleurs au Québec. Perspectives interdisciplinaires sur le travail et la santé, 4-2. http://pistes.revues.org/3661; DOI : 10.4000/pistes.3661.

Bessy, O., \& Chateauraynaud, F. (1995). Experts et faussaires. Pour une sociologie de la perception. Paris : Métailié.

Boltanski, L. (1990). L'amour et la justice comme compétence. Trois essais de sociologie de I'action. Paris : Métailié.

Boltanski, L., \& Thévenot, L. (1991). De la justification. (1è éd. 1987). Paris : Gallimard.

Caroly, S., Simonet, P., \& Vézina, N. (2015). Marge de manœuvre et pouvoir d'agir dans la prévention des TMS et des RPS. Le travail humain, vol. 78, 1, 1-8.

Coutarel, F. (2005). Développer les marges de manœuvre et évaluer nos interventions pour faire face aux TMS : quelles conditions à une prévention durable ? 40e Congrès de la SELF, France. pp. 132-139.

CNFPT (2014). La prise en compte de la pénibilité au travail dans les collectivités territoriales. Repéré à http://cnfpt.fr/s-informer/etudes/prise-compte-penibilite-au-travail$\mathrm{fpt} /$ national?gl=NzdhNjlmMmQ.

Desbarat, I. (2010). Obligation de reclassement et obligation de sécurité : quelle articulation en matière de santé au travail ? Perspectives interdisciplinaires sur le travail et la santé, 12-1. http:// pistes.revues.org/1571; DOI : 10.4000/pistes.1571.

DARES (2015). Le devenir professionnel des actifs en mauvaise santé, 68. Repéré à http://dares.travail-emploi.gouv.fr/IMG/pdf/2015-068.pdf.

DIRECCTE Aquitaine (2015). Inaptitude médicale totale et définitive à tous postes dans l'entreprise. Enquête 2014 Aquitaine.

DIRRECTE Pays de Loire (2012). L'inaptitude en 50 questions. Repéré à http://travailemploi.gouv.fr/IMG/pdf/Inaptitude-en-50_questions-2_edt_12-2012.pdf.

Dodier, N. (1986). Corps fragiles. La construction sociale des événements corporels dans les activités quotidiennes du travail. Revue française de sociologie, vol. 27, 4, 603-628. 
Dubet, F. (2006). Injustices. L'expérience des inégalités au travail. Paris : Seuil.

Dubost, J. (1987). L'intervention psychosociologique. Paris : PUF.

Dujarier, M.-A., Gaudart, C., Gillet, A., \& Lenel, P. (Eds) (2016). L'activité en théories, regards croisés sur le travail. Toulouse : Octarès.

Fantoni, S. (2016). Étendue et limites de l'obligation de reclassement à l'égard des personnes présentant une inaptitude en France, Perspectives interdisciplinaires sur le travail et la santé, 12-1. http:// pistes.revues.org/1603 ; DOI : 10.4000/pistes.1603.

Fantoni-Quinton, S., \& Frimat, P. (2011). Maintien au travail et maladie : un exemple d'accompagnement individualisé en région Nord. Santé Publique, vol. 23(3), 251-259. DOI : $10.3917 /$ spub.113.0251.

FNCDG, (2015). Le reclassement pour inaptitude physique. De la procédure juridique à la mise en œuvre. En collaboration avec l'UNCCAS. Repéré à http:// www.handipole.org/IMG/pdf/-78.pdf.

Gaillard, I., \& de Terssac, G. (2013). Compétences organisationnelles et santé. Sociologies pratiques, 26, 19-26.

Gollac, M., \& Volkoff, S. (2000). Les conditions de travail. Paris : La Découverte.

Haïlé-Fida, S. (2005). Quelle place pour la maîtrise de proximité dans la détection et la prise en charge de l'usure professionnelle ? Le cas d'une société de transports urbains de voyageurs de la région Rhône-Alpes, La Revue de I'Ires, 48, 101-125. Repéré à http://www.ires-fr.org/publications-de-l-ires/item/2653-quelle-place-pour-la-maitrisede-proximite-dans-la-detection-et-la-prise-en-charge-de-l-usure-professionnelle-lecas-d-une-societe-de-transports-urbains-de-voyageurs-de-la-region-rhone-alpes.

Héas, F. (2010), Pénibilité au travail : reclasser ou prévenir ? Perspectives interdisciplinaires sur le travail et la santé, 12-1, http://pistes.revues.org/1664; DOI : 10.4000/pistes.1664.

Huyez-Levrat, G., \& Waser, A.-M. (2014). L'avantage mutuel. Recherche-action sur le retour et le maintien en activité de salariés touchés par une maladie chronique. La nouvelle revue du travail, 4. http://nrt.revues.org/1441.

Krynen, B., \& Yeni, I. (2011). Evaluation du dispositif de reclassement des fonctionnaires déclarés inaptes à l'exercice de leurs fonctions pour des raisons de santé (Publication $\mathrm{n}^{\circ}$ RM2011-174P, IGAS). Repéré sur le site de La documentation française. http://www.ladocumentationfrancaise.fr/var/storage/rapports-publics/124000208.pdf

Laé, J.-F. (1991). L'inaptitude à la RATP, de la protection à la sanction. Sociétés contemporaines, 8, 107-125.

Le Bianic, T. (2003). L'inaptitude : une construction sociale ? Education permanente, 156, 5566.

Lecomte-Ménahès, G. (2014). Santé précaire et transformation de la médecine du travail. Travail et Emploi, 140, 35-47.

Leplat, J. (2008). Repères pour l'analyse de l'activité en ergonomie. Paris : PUF.

Leplat, J. (2006). La notion de régulation dans l'analyse de l'activité, Perspectives interdisciplinaires sur le travail et la santé, 8-1. http://pistes.revues.org/3101; DOI : 
10.4000/pistes.3101.

Lhuilier, D. (2017). La recherche-action en psychosociologie du travail. In A.-L. Ulmann, A. Weill-Fassina, \& T. H. Benchekroun (Eds), Intervenir. Histoires, recherches, pratiques (pp. 63-74). Toulouse : Octarès.

Lhuilier, D. (2006). Cliniques du travail. Paris : Erès.

Lhuilier, D. (2002). Placardisés. Paris : Seuil.

Lhuilier, D., \& Waser, A.-M. (2016). Que font les 10 millions de malades ? Vivre et travailler avec une maladie chronique. Toulouse : Erès.

Lhuilier, D., Waser, A.M., Mezza, J., \& Hermand, C. (2014). Restauration de la puissance d'agir par un travail en groupe : retour sur les fonctions et transformations des « clubs Maladies Chroniques et Activité ». Pratiques psychologiques, 20(4), 249-263. http://dx.doi.org/10.1016/j.prps.2014.09.001.

Maresca \& Dujin, (2011). Le maintien dans l'emploi des salariés ayant connu la longue maladie. Quelle place dans les stratégies des grandes entreprises en France et en Allemagne ? Pratiques et Organisation des Soins, 42(1), 19-26.

Mendel, G., \& Prades, J.-L. (2002). Les méthodes de l'intervention psychosociologique. Paris : La Découverte.

Mezza, J. (2017). Les pratiques d'orientation en direction des personnes malades chroniques, Perspectives interdisciplinaires sur le travail et la santé, 19-2. http://pistes.revues.org/5093 ; DOI : 10.4000/pistes.5093

Molinié, A.F., \& Pueyo, V. (2012). Les dynamiques temporelles des relations santé et travail et le fil de l'âge. In A.-F. Molinié, C. Gaudart, \& V. Pueyo (Eds.) La vie professionnelle : âge, expérience et santé à l'épreuve des conditions de travail. Toulouse : Octarès Editions

Obrecht, O., \& Hittinger-Le Gros M.-C. (2010). Maladies chroniques et travail : au-delà des idées reçues. Paris : Editions de Santé et Presses de Sciences Po.

Olry-Louis, I., Vonthron, A.-M., Vayre, E., \& Soidet, I. (2017). Les transitions professionnelles. Nouvelles problématiques psychosociales. Paris : Dunod.

Petit, J., \& Dugué, B. (2013). Quand l'organisation empêche un travail de qualité : étude de cas. Perspectives interdisciplinaires sur le travail et la santé, 15-2. http://pistes.revues.org/3419; DOI : 10.4000/pistes.3419

Pommier, J.-L., Bardouillet, M.-C., Gilles, M., \& Molinié, A.-F. (2006). Ce salarié devrait cesser de travailler: une approche de l'usure professionnelle des 50 ans et plus. Retraite et société, 49, 39-59.

Sarnin, P., Bobillier-Chaumon, M.-E., Cuvillier, B., \& Grosjean, M. (2012). Intervenir sur les souffrances au travail : acteurs et enjeux dans la durée. Bulletin de psychologie, Tome 65 (3), 519, 251-262.

de Terssac, G. (2008). Le travail et la santé : une question d'organisation ? In G. de Terssac, C. Saint-Martin, \& C. Thébault (Eds), La précarité : une relation entre travail, organisation et santé (pp. 209-226). Toulouse : Octarès.

de Terssac, G. (1992). Autonomie dans le travail. Paris : PUF. 
Veil, C. (2012). Vulnérabilités au travail. Naissance et actualité de la psychopathologie du travail. Toulouse : Erès.

Volkoff, S. (2015). Les autres « pénibilités ». Fragilisation de la santé, et vécu du travail en fin de vie active. Retraite et société, vol. 72(3), 87-101. 